\title{
Olivier Thibodean
}

\section{Des pièces de go sur l'échiquier: Vitesse(s) et mouvement(s) révolutionnaire(s) dans le film de zombies contemporain}

\section{Go Stones on the Chessboard:}

\section{REVOLUTIONARY VELOCITY IN CONTEMPORARY}

\section{ZOMBIE FILMS}

Abstract: The zombie as metaphor for the mindless consumer of late capitalism, popularized by George Romero's seminal masterpiece, Dawn of the Dead, has grown stale as a critical model for globalized capitalism in light of its power not to embrace, but to disrupt the dromocratic order in which capitalism is rooted. If it is fair to assimilate the zombie mass to the proletarian class freed from the shackles of "productive" labor, it is of greater importance today to address its freedom from the whole kinetic organization of life under capitalist state rule.

Keywords: Zombie; Cinema; Dromocracy; Speed; Proletariat; Revolution; Paul Virilio; Gilles Deleuze.

\section{OLIVIER THIBOdEAU}

Université de Montréal, Montréal, Canada olivier.tj13@gmail.com

DOI: 10.24193/cechinox.2020.38.09

\section{Introduction : le prolétariat}

\section{libéré}

$\ll \mathbf{W}_{\text {of scholarship concerning Dawn }}^{\text {of } \text { Dead [1978] focuses on the }}$ film's rather overt criticism of contemporary consumer culture " ${ }^{1}$, résume Kyle William Bishop dans l'introduction de son article, The Idle Proletariat, qu'il dédie à l'analyse du discours académique entourant le film de Romero. "The metaphor is simple: Americans in the 1970s have become a kind of zombie already, slaves to the master of consumerism, and mindlessly migrating to the malls for the almost instinctual consumption of goods $»^{2}$. Cette idée d'automate décervelé, mu par les pulsions motrices issues $\mathrm{du}$ mode de vie consumériste, a fait beaucoup de chemin déjà dans l'arène académique, mais il constitue finalement une impasse, au vu du caractère historiquement évolutif et du potentiel révolutionnaire de la figure du zombie, mais surtout de la logique politique voulant que, contrairement au zombie, le consommateur décervelé ne constitue jamais une menace pour l'ordre établi. Bishop pousse la réflexion plus à fond en suggérant que c'est la perte de dessein individuel associé au système consumériste que 
représente en fait la figure du zombie, puisant dans la théorie marxiste voulant que ce soit le travail productif qui donne sens à l'existence humaine, évoquant «l'oisiveté du prolétariat » comme cause de son errance. J'arguerai a contrario que cette forme d'oisiveté constitue en fait une libération pour la masse prolétaire zombie, dont les mouvements ne sont plus régis par les impératifs bourgeois de production ou par la logique dromocrate défendue par les bâtisseurs du système étatique mondial et leurs équivalents diégétiques, les « survivants » humains qui leur servent d'ennemis.

Bishop touche déjà au nœud du problème lorsqu'il décrit à leur tour les quatre protagonistes de Dawn of the Dead comme " having been essentially brainwashed by capitalist ideology. They cannot see the shattered world around them in any other than those of possession and consumption. ${ }^{3} \mathrm{Il}$ assimile ainsi la figure du zombie et celle du survivant en tant que sous-produits communs $\mathrm{du}$ système capitaliste. C'est du moins la base de son propos, qui se désintéresse ensuite peu à peu des zombies au profit des survivants et de la perte de sens que représente leur privation de travail productif. Nonobstant son adéquation contentieuse du travail policier avec le travail dit " productif » qu'il associe ailleurs au prolétariat, il assimile en outre de façon problématique la manière de consommer des survivants à celle des zombies, qu'il décrit, via A. Loudermilk, comme nos "simulacral doubles as cannibal consumers " ${ }^{4}$, ignorant ce faisant l'assertion diégétique à l'effet que les zombies ne sont pas des cannibales puisqu'ils ne se dévorent jamais entre eux. Or, l'évidence semble prouver le contraire, c'est-à-dire que les deux groupes pratiquent en fait deux modes de consommation parfaitement distincts : la consommation de survivance des zombies, qui ne mangent finalement que pour survivre, et la consommation pillarde des survivants, qui s'approprient et conservent jalousement toutes les ressources à leur disposition selon un ethos colonialiste complètement étranger à l'étiquette de leurs contreparties.

Selon Dan Hassler-Forrest (cité par Sarah Julliet Lauro), le récit de zombie contemporain typique s'inspire de la formule héritée de Robinson Crusoé, où les survivants préservent l'éthos colonialiste en œuvrant à dévaliser les ressources terrestres disponibles ${ }^{5}$. Le terme "préserver » est essentiel ici, puisqu'il implique une forme de pérennité dans l'exercice du pouvoir bourgeois que représentent les survivants en question. En effet, si les instincts résiduels des zombies les amènent à fréquenter les hauts lieux du consumérisme, il est à noter qu'ils n'y consomment ou n'y pillent finalement rien, outre la chair humaine qu'ils partagent entre eux - les scènes de repas « communautaires » tendant en effet à prouver leur nature collégiale. Plutôt que de constituer les rouages de la société consumériste, ils en sont en fait les mécanismes antagonistes. Non seulement ils ne consomment plus rien (outre les survivants colonialistes), mais ils résistent parfaitement au processus d'individualisation sous-jacent au système capitaliste. Les zombies de Dawn of the Dead, comme ceux de tous les exemples contemporains du genre, ne sont pas des esclaves de ce système, mais bien des parvenus. Ils sont libres, anonymes mais surtout, ils sont concertés. Ils sont la masse prolétaire libérée des impératifs de l'action productive, et 
en cela les protagonistes d'un récit où le rôle d'oppresseur est joué par leurs contreparties humaines, attachées mordicus à l'exercice d'un pouvoir égoïste axé sur la prise de possession et la garde jalouse des ressources terrestres, mais surtout sur la réinstauration du contrôle des mouvements de la meute zombie.

Bishop touche d'ailleurs encore au nœud du problème lorsqu'il évoque la cupidité desdits protagonistes, qui refusent de partager leurs cigarettes avec d'autres survivants et qui affrontent les pilleurs à moto du climax pour la possession des ressources acquises militairement dans le centre d'achats. Il évoque même de façon explicite le rapport colonialiste qui lie les deux groupes lorsqu'il déclare que " the movie reestablishes the zombies as pathetic metaphors for colonial native peoples: the humans have arrived to invade and plunder an existing, exotic location, securing the borders before wiping ou the 'indigenous population' in a bloobath of reckless violence ${ }^{6}$. Notons à cet effet qu'au-delà de la mainmise des ressources, les protagonistes imposent ici une gestion de l'espace intrinsèquement policière : elle consiste à évincer tous les zombies du centre d'achats où ils déambulent, à en bloquer l'accès avec des semi-remorques et à contrôler cet accès l'arme au poing, bref à ré-instituer le contrôle bourgeois du mouvement prolétaire qu'avait su dérégler la révolte zombie. Le pouvoir des protagonistes est intrinsèquement dromocratique, pour reprendre l'expression de Paul Virilio, c'est-à-dire qu'il repose sur la gestion de la voirie, tandis que le pouvoir zombie est anti-dromocratique, c'est-à-dire libre (ou plutôt libéré) de toute embâcle ambulatoire. Et c'est là précisément que réside l'intérêt politique du film de zombies : dans la friction constante entre l'attachement sentimental de la bourgeoisie survivante aux mécanismes dromocratiques colonialistes et la liberté de mouvement du prolétariat émancipé que représente la masse zombie, intérêt politique qui dépasse d'ailleurs largement le cadre du cinéma de Romero pour inclure toute l'histoire du genre et permet de réhabiliter le zombie comme figure révolutionnaire dans une perspective de transgressivité planétaire, de lutte au pouvoir étatique, de lissage des espaces géopolitiques, bref d'annihilation des frontières physiques jalousement policées par le pouvoir dromocrate au profit d'un espace nomade mondialisé.

\section{Potentiel révolutionnaire de la vitesse zombie}

C'est un an seulement avant la sortie de Dawn of the Dead que Paul Virilio publiait Vitesse et politique, qui depuis pourrait être considéré comme le manifeste définitif du cinéma zombie. Notons d'abord le parallèle appuyé que trace l'auteur entre la masse prolétaire et la meute animale, ce " cheptel » qu'il importe de dresser et de contrôler afin d'empêcher sa férocité potentielle de s'exprimer contre ses maîtres. Dans son analyse sur l'évolution de la banlieue, Virilio déclare notamment que celle-ci est «à la fois juridiction d'interdit et distance linéaire et horaire, c'est-à-dire dépôt et rupture de charge de la matière sociale comme des marchandises, des vivres et de ce bétail auquel le prolétariat 'brassier' est assimilé depuis des millénaires, animaux plus ou moins sauvages devenus bêtes de somme, de guerre ou de bât; les conditions d'exploitation 
des masses prolétarisées illustre d'ailleurs parfaitement la définition que Geoffroy Saint-Hilaire donne à la domestication : 'domestiquer un animal, c'est l'habituer à vivre et à se reproduire dans les demeures des hommes ou auprès d'elles' $»^{7}$. La masse des travailleurs donc, c'est la meute domestiquée, celle qui se meut selon des schèmes préétablis, selon une logique d'État fasciste qui en l'empêchant systématiquement de se regrouper ou de se mouvoir sans autorisation dans les rues, mine dans l'œuf tout son potentiel révolutionnaire. En effet, si le contrôle étatique du mouvement peut s'effectuer via de nombreux dispositifs, il réside surtout chez Virilio dans la supervision de la voirie, et c'est là particulièrement que ses théories recoupent l'iconographie récurrente du cinéma des zombies.

«Le pouvoir politique de l'État n'est que secondairement le pouvoir organisé d'une classe pour l'oppression d'une autre ", déclare Virilio, " plus matériellement, il est polis, police, c'est-à-dire voirie et ceci dans la mesure où, depuis l'aube de la révolution bourgeoise, le discours politique n'est qu'une série de prises en charges plus ou moins conscientes de la vieille poliorcétique communale, confondant l'ordre social avec le contrôle de la circulation [...] et la révolution, l'émeute, avec l'embouteillage, le stationnement illicite, le carambolage, la collision $»^{8}$. Et c'est d'ailleurs là que réside « la menace, la charge d'inconnu et de férocité $»^{9}$ que porte en elle la meute prolétaire, et qui justifie sa domestication par l'institution bourgeoise, menace qui implique le potentiel de démanteler le pouvoir de la voirie, c'est-à-dire de transcender le pouvoir de coercition sociale qu'implique le contrôle du mouvement, de reprendre la rue aux policiers, et d'abattre les barrières qui servent de limites au mouvement humain. C'est le potentiel pour la meute prolétaire de briser le carcan capitaliste en sémancipant des lieux de production et en se libérant du contrôle horaire/moteur quelle y subit. "La masse ", dit Virilio, « n'est pas peuple ou société », mais « multitude $»^{10}$, « multitude des corps sans âmes, morts-vivants, zombies, possédés, etc. ${ }^{11}$, rappelant ainsi la masse indifférenciée des zombies cinématographiques, qui elle aussi atteint son plein potentiel révolutionnaire en devenant productrice de vitesse, c'està-dire en libérant sa vitesse de l'esclavage horaire/moteur inhérent au système capitaliste. Notons à cet égard que la prise de vitesse historique de la figure du zombie correspond à l'accélération de la machine responsable de son assujettissement, mais surtout de la peur qu'implique son potentiel grandissant de libération auprès des systèmes autoritaires mondiaux.

L'essor du mouvement de masse prolétaire que représente la révolte zombie se mesure de façon particulièrement évocatrice dans les gradations de vitesse historiques de la figure, lesquelles représentent en fait sa liberté grandissante, plutôt que sa servitude face à l'institution étatico-capitaliste détentrice du pouvoir dromocrate. À ses débuts, le zombie cinématographique est d'origine vaudou. C'est un travailleur décervelé, doté de nulle autre volition que celle du maitre. C'est l'équivalent de l'esclave noir dans les États-Unis de l'avant-guerre civile, ouvrier domestiqué par peur de représailles dont chacun des mouvements est scrupuleusement contrôlé afin de prévenir toute organisation révolutionnaire. Ce n'est donc pas un 
hasard si les zombies cinématographiques de la première époque se retrouvent dans des plantations caribéennes dirigées par des Blancs, comme dans le White Zombie (1932) de Victor Halperin ou le $I$ Walked with a Zombie (1943) de Jacques Tourneur. C'est qu'il s'agit de l'équivalent métaphorique spéculaire de l'esclave noir des champs de coton étasunien, un travailleur docile à la vitesse révolutionnaire nulle. D'ailleurs, force est de constater que l'image emblématique du film de Tourneur est celle du zombie stationnaire à l'orée des champs, le zombie statique au regard vide dont la motricité n'est tributaire que des besoins de son maître.

Tout change en 1968, avec l'avènement des " morts » conçus par George Romero, ces zombies mouvant de leur propre volition, aléatoirement, indépendamment des pouvoirs esclavagistes et étatiques, destructeurs des systèmes de voiries et repoussoirs de la bourgeoisie ambulante dans ses propres retranchements selon une logique de sédentarité forcée qu'ils érigent alors en parodie. C'est la preuve de ce qu'avance le colonel Delair lorsqu'il déclare que "le stationnement, c'est la mort ${ }^{12}$. Seule la vitesse et le mouvement peuvent en effet assurer la survie humaine dans le cinéma de Romero. C'est ce que prouve la fin de Night of the Living Dead, alors que les gardiens du château meurent tous aux mains des gardiens ambulants de la voirie, la milice qui tente coûte que coûte de reprendre le contrôle des mouvements de population. C'est ce que prouve également la fin de Dawn of the Dead, où l'idylle bourgeoise des protagonistes est détruite de vitesse par le groupe de pilleurs à moto, et où la seule échappatoire est de «battre » de vitesse ces derniers grâce au transport héliporté. Ce qu'il y a de plus intéressant encore dans la tétralogie des "morts " de Romero, c'est qu'elle emblématise à elle seule la tendance historique du film de zombie à toujours rapprocher ce dernier de la source du pouvoir dromocratique, c'està-dire l'institution étatique et militaire. On note ainsi que de l'univers rural de Night, on passe à l'univers banlieusard de Dawn, puis à l'univers urbain de Day of the Dead (1985), où les zombies parviennent même à anéantir l'institution militaire, gardienne de la voirie. Finalement, avec Land of the Dead (2005), c'est l'État lui-même qui finit par tomber, du moins cet ersatz d'État que représente la ville fortifiée de Fiddler's Green où règne le Kaufman de Dennis Hopper, alter-ego évident du George Bush de l'après 11 septembre. De manière significative, c'est d'ailleurs en passant $\grave{a}$ travers la rivière que la meute parvient aux portes de la Cité, c'est-à-dire en prenant le contrôle des espaces lisses, des espaces « libres » situés en périphérie.

La vitesse exponentielle de la meute dans les films de zombies récents, ainsi que leur dispersion géopolitique massive suggèrent une mainmise encore accrue sur la voirie, un contrôle mondial en fait, qui entraîne une destruction totale des frontières, un lissage pour ainsi dire complet des espaces striés par l'État et l'institution militaro-bourgeoise. Si c'est le cas dans 28 Weeks Later (2007), où la cité militaire fortifiée du récit finit par tomber sous le poids de la meute, c'est encore plus évident dans World War Z (2013), où on met en scène la destruction d'Israël, état emblématique du contrôle de la voirie et des flux migratoires, société de sas où la discipline militaire 
englobe toute la population, détruite ici totalement par la fluidité de la masse lancée vers elle, destructrice de murs, et instigatrice d'un monde nouveau. On pourrait même dire que World War $Z$ est un film axé exclusivement autour des enjeux de voirie et de vitesse véhiculaire. Ce n'est pas un hasard en effet si l'invasion diégétique des États-Unis commence au moment où les protagonistes sont coincés dans un embouteillage, c'est-à-dire où ils sont ironiquement immobilisés par leur propre système de contrôle des masses, et où ils doivent combattre la meute de vitesse, via l'utilisation de véhicules automobiles, puis héliportés et turbo-propulsés. Si le film s'intéresse surtout à la déambulation du protagoniste, des États-Unis vers la Corée, puis vers Israël, le Royaume-Uni et le Canada à la manière d'un James Bond du cinéma d'horreur, il s'intéresse surtout au mouvement des zombies, à la fluidité de ce mouvement, fluidité telle qu'elle leur permet éventuellement de contrôler la terre et les airs, mais surtout d'abattre les frontières qui servent chez Virilio de "barrages", de " filtres à la fluidité des masses ", à la «puissance de pénétration $»^{13}$ des meutes migratrices.

\section{Pouvoir de pénétration de la meute et panique institutionnelle}

Si la figure du zombie évoque, via ses gradations historiques de vitesse, le potentiel grandissant de l'émancipation prolétaire mondiale face au contrôle cinétique dromocrate, il représente subséquemment une menace grandissante pour cet ordre étatique aujourd'hui paniqué, obsédé plus que jamais par le contrôle des flux migratoires, par la traque électronique des individus et par la sécurité frontalière face, notamment, à l'avancée des masses « barbares » méridionales vers le Nord. À ce titre, il est intéressant de noter comment le scénario, voire l'iconographie entière du film de zombies s'est resserrée historiquement autour des enjeux de voirie qui forment désormais le cœur simultané du genre et des stratégies politiques d'État réelles. La tétralogie des morts de Romero proposait déjà un survol du parcours historique suivi par le zombie, de la périphérie du monde civilisé vers le cœur de la cité, mais ce parcours mérite encore d'être détaillé, particulièrement au vu du changement de paradigme qui caractérise le genre dans l'après-11 septembre.

Lors de son apparition dans le cinéma des années 30-40, la figure du zombie constitue surtout une menace " exotique " (à l'instar des peuples sauvages du serial d'aventure ou du péril jaune à la Fu-Manchu). Elle est alors presque exclusivement confinée aux repaires insulaires de la bourgeoisie colonialiste, comme dans White Zombie, Ouanga (1936), Revolt of the Zombies (1936), King of the Zombies (1941), I Walked with a Zombie (1943), Revenge of the Zombies (1943), Voodoo Man (1944), et même Zombies on Broadway (1945); la terminologie titulaire évoque déjà pourtant une forme d'insurrection (revolt, revenge) face à l'institution bourgeoise. Cette perspective exotique sur la figure demeure largement inchangée jusqu'aux années 80 . Exception faite de la pollinisation exercée par le cinéma de science-fiction catastrophique, qui en fait une menace globale affiliée aux extra-terrestres (comme dans Invisible Invaders [1959], Plan 9 From Outer Space [1959] ou The Astro-Zombies [1968]), le zombie reste associé au monde 
fantastique de la sorcellerie païnne (Voodoo Island [1957], Zombies of Mora Tau [1957], War of the Zombies [1964], Tombs of the Blind Dead [1971], Return of the Blind Dead [1973], Shock Waves [1977]). C'est au tournant des années 80, dans le sillon de Dawn of the Dead qu'il commence à vraiment investir l'espace suburbain, conséquemment avec l'avènement du cinéma d'horreur banlieusard qui devait caractériser la production étasunienne de la fin du siècle. S'il commence à menacer l'humanité, c'est surtout localement, comme dans City of the Living Dead (1980), The Fog (1980), Dead E Buried (1981), Zombie Lake (1981), Mutant (1984), The Return of the Living Dead (1985), Night of the Creeps (1986), Redneck Zombies (1987) et The Dead Next Door (1988). Il s'agit néanmoins d'une avancée significative pour la meute, qui quitte les domaines excentrés et les lieux de villégiature de la bourgeoisie dromocrate, et commence à perturber l'ensemble de ses mouvements quotidiens.

Ce n'est qu'au tournant du millénaire que le zombie constituera une menace globale, avec pour triple assise l'exploitation désormais industrielle du genre, le scénario opportun du «zombie » viral et, l'un de ses corollaires, le scénario politique de l'invasion «barbare ». Force est effectivement de constater qu'à la fin des années 90 , la figure du zombie se libère de ses origines surnaturelles, et devient principalement pandémique (voir notamment Plaga Zombie [1997], Bio Zombie [1998] et Biohazardous [2001]). Le manifeste du nouveau cinéma zombie se trouve pourtant cristallisé dans le 28 Days Later (2002) de Danny Boyle, où non seulement la meute des «infectés " s'attaque au cœur même de la civilisation occidentale (Londres, noyau historique de l'institution dromocrate colonialiste), mais où sa vitesse commence à s'accentuer de façon dramatique. D'un côté la peur de l'infection évoque alors les angoisses médicales de la société pharmacologique contemporaine, mais la vélocité accrue du zombie (qui court désormais à toutes jambes vers ses proies) évoque quant à elle la peur que représente le potentiel de pénétration accru de la meute «barbare » dans l'imaginaire occidental de l'après 11 septembre. Il est crucial en effet de noter qu'avant de constituer une victoire contre l'impérialisme économique des " païens " étasuniens, les attaques du 11 septembre 2001 constituent une victoire contre leur système de voirie, telle qu'exemplifiée par le détournement des avions « de ligne » utilisés par les Djihadistes, c'est-àdire par leur réappropriation antagoniste du contrôle cinétique dromocratique. À bien des égards, lévénement en question marque le début de ce que les pouvoirs occidentaux envisagent comme les « invasions barbares " (pour reprendre l'expression un peu maladroite de l'ex-historien Denys Arcand dans son film éponyme de 2003) : invasion fabulatoire de la doctrine djihadiste d'une part, et invasion subséquente de toutes les "violences étrangères " (que représentent notamment les « violeurs » et « les méchants " mexicains de Donald Trump). Dans l'imaginaire populaire, la prolifération de la doctrine sectariste du Djihad, mais aussi la progression septentrionale des caravanes de migrants africains et sud-américains revêt désormais un caractère viral, à l'instar de la figure du zombie avec laquelle elle partage un "pouvoir de pénétration » renouvelé. Le zombie est viral certes, mais il est en fait complètement assimilable au virus, en ce sens qu'il constitue une menace transnationale incloisonnable, à l'instar du 
barbare qui envahit l'espace aérien étasunien ou l'espace maritime méditerranéen.

À la première époque du cinéma des zombies (1932-1968), le pouvoir de pénétration de la meute est quasi-nul en ce sens qu'elle déborde rarement les frontières des mondes exotiques où elle est confinée, en ce sens que la meute n'est même pas encore la meute, mais une poignée d'esclaves inorganisés. Et si ces derniers font embâcle à la poussée colonialiste des puissances occidentales, ce n'est qu'en pénétrant les quelques avant-postes excentrés qu'elles possèdent à la périphérie de leur propre territoire. Le pouvoir de pénétration de la meute zombie s'accroit à la deuxième époque (19682002), alors que celle-ci atteint l'intérieur des terres, où elle commence à perturber les mouvements dromomanes des peuples. Les zombies obstruent alors la voie des protagonistes, et compromettent leurs mouvements quotidiens; ils se mettent à contrôler la voirie, mais d'une façon libre, d'une façon fluide, sans recours aux diktats légaux ou à l'organisation martiale de l'institution bourgeoise, qui commence dès lors à les combattre pour reprendre son dû. À la troisième époque (2002-), son pouvoir de pénétration est devenu mondial ; les frontières physiques autrefois impénétrables deviennent poreuses (la Manche qui semblait protéger le continent européen des îles britanniques faillit à la tâche dans 28 Weeks Later et même le mur à la frontière israélo-palestinienne cède dans World War Z). C'est un lissage complet de l'univers strié par les institutions dromocrates auquel on assiste désormais, une victoire de ce que Deleuze et Guattari pourraient qualifier la « machine de guerre » zombie.

Le cinéma de masse étant généralement destiné à la défense du statu quo (celui-ci représentant dans le scénario hollywoodien classique la situation initiale à laquelle il importe de retourner à la fin du récit), on propose subséquemment au spectateur de s'identifier aux " héros " du stato quo (le personnage de T'Challa dans Black Panther [2018], par exemple alors que c'est l'antagoniste N'Jadaka qui constitue la vraie force de changement, et le vrai «black panther ", étiquette politique dont les studios se réapproprient ainsi la signification réelle). Dans le cinéma de zombies, cela signifie que c'est toujours le survivant chez qui le spectateur est censé se retrouver, et c'est le zombie qu'il doit abhorrer pour son pouvoir de disruption du statu quo. À ce titre, force est de constater que c'est désormais en mode panique que le cinéma de masse s'oppose à la révolte des zombies, disséminant une conception de plus en plus catastrophique de leur prise de pouvoir, c'est-à-dire du remplacement de l'institution fasco-démocratique actuelle par les forces anarchistes. Dans l'ère post-11 septembre, les choses s'accélèrent énormément, et les révolutionnaire nomades, les révolutionnaires barbares de la meute deviennent littéralement enragés. Ils se mettent à courir et les enjeux de voirie deviennent dantesques. C'est aussi là que se cristallisent les mécanismes pervers d'identification propres au genre, où plutôt que de s'identifier à la masse prolétaire zombie, la masse prolétaire des spectateurs doit s'identifier aux survivants, représentants de la bourgeoisie sédentaire, attachés au pouvoir étatique comme à la servitude volontaire que représente son adhésion. C'est le cas de la série Walking Dead, où lorsqu'ils ne sont pas en quête de l'État lui-même (le CDC par exemple, ou le Capitole), les protagonistes s'accrochent obstinément à 
ses chasses-gardées, la prison par exemple, ou la ferme d'Herschell, sanctuaires de l'existence sédentaire réglée par le pouvoir patriarcal traditionnel. Les protagonistes survivants, comme attachés de façon nostalgique à un pouvoir d'état familièrement fasciste, œuvrent presque systématiquement à sa reconstruction, incapables de s'adapter, même de s'imaginer vivre dans le monde lisse, le monde lissé par les nomades. Leur difficulté de mouvement vient d'ailleurs du fait qu'ils se meuvent d'une façon incompatible avec la nature même du nouvel ordre mondial. Pour reprendre le fil de la réflexion deleuzo-guattarienne, on dira qu'il s'agit pour ainsi dire de pièces d'échec tentant d'évoluer sur un plateau de go.

\section{La machine de guerre zombie}

$\mathrm{P}$ our mieux comprendre la nature de l'organisation révolutionnaire zombie, il importe désormais d'évoquer le texte corollaire du Vitesse et politique de Virilio, soit le " traité de nomadologie " de Mille Plateaux qui, comme celui de dromologie avant lui, nous permet de cerner la nature antiétatique de l'action zombie, assimilable à celle des guerriers nomades de la théorie deleuzo-guattarienne, dont on décrit l'organisation comme celle d'une machine de guerre non-militaire, non-étatique. En effet, si Virilio parle de la machine de guerre comme de la meute militarisée, les auteurs de Mille Plateaux voient plutôt celle-ci échapper au contrôle de l'État, lui entrevoyant même une fonction spécifiquement antiétatique. La machine de guerre provient d'ailleurs selon eux, à l'instar du zombie exotique, du zombie envahisseur, du zombie viral. Elle est multiplicité pure et sans mesure, la meute ; elle est célérité contre gravité. Leur comparaison opératoire consiste d'ailleurs à assimiler l'appareil d'État à l'échiquier, et la machine de guerre qui lui est opposée, à la planche de go, les pièces correspondant aux planches respectives pouvant être identifiées par la discipline, et l'indiscipline qui caractérise leurs mouvements respectifs, à l'instar des différents groupes en présence dans le cinéma de zombies contemporain (soit les survivants, dédiés au mimétisme de la logique d'État, et la meute révolutionnaire zombie). De façon plus révélatrice encore, ils assimilent l'espace étatique tout entier à un espace strié, un espace sédentaire, et la machine de guerre à l'espace lisse du nomade.

Selon les deux auteurs, l'un des pouvoirs de la machine de guerre non-étatique est justement de lisser l'espace, et c'est là quon peut imaginer la meute, la meute des zombies, mais avant elle l'organisation de bande pré-étatique. Si l'organisation pré-étatique de la meute inhibe intrinsèquement le pouvoir d'État, c'est justement parce qu'elle est basée sur une série de relations immanentes, parce quelle est rbizomateuse, "par opposition au type arborescent qui se concentre sur des organes de pouvoir ${ }^{14}$. C'est la nature même du pouvoir zombie, qui n'est jamais centralisé, et dont l'extermination est conséquemment si ardue, contrairement au pouvoir vampire par exemple, qui lui est hiérarchique, et centralisé sur la figure du vampire "premier ", source radicale d'une arborescence dont il suffit alors d'éliminer le tronc. Le caractère itinérant du zombie constitue lui aussi une forme d'opposition au pouvoir étatique. "On sait les problèmes que les États ont toujours eus avec les 
Vitesse(s) et mouvement(s) révolutionnaire(s) dans le film de zombies contemporain

'compagnonnages', les corps nomades ou itinérants de type maçons, charpentiers, forgerons ", déclarent Deleuze et Guattari, «Fixer, sédentariser la force de travail, régler le mouvement du flux de travail, lui assigner des canaux et conduits, faire des corporations au sens d'organismes, et, pour le reste, faire appel à une main-d'œuvre forcée, recrutée sur les lieux ou chez les indigents - ce fut toujours une des affaires principales de l'État, qui se proposait à la fois de vaincre un vagabondage de bande, et un nomadisme de corps $»^{15}$. Au même titre que l'État a pour tâche fondamentale de strier l'espace pour y asseoir son pouvoir, le nomade effectue par nature l'inhibition de l'État via le lissage de ses stries. "Le nomade fait lui-même croître les lieux lisses au sens où l'on constate que le nomade fait le désert non moins quill est fait par lui. Il est vecteur de déterritorialisation. ${ }^{16}$

Mais pourquoi exactement la forme étatique a-t-elle triomphé sur le modèle d'organisation en «bandes » qui dominait avant son avènement ? C'est ce que posent aussi comme question Deleuze et Guattari. Serait-ce que la pensée est déjà conforme à un modèle qu'elle emprunterait à l'appareil d'État, et qu'elle ne puisse en somme que légitimer l'État et le recréer partout ? C'est du moins ce que semble suggérer le modèle d'identification cinématographique discuté précédemment, qui, sauf quelques exemples rebelles, contraint le spectateur à se borner à la nostalgie étatique des survivants du film de zombies. En effet, force est de constater que ceux-ci ne peuvent jamais échapper au modèle de pensée étatique, et ce bien qu'il existe une façon d'être alternative, un moyen pour eux de se fondre à la meute nomade des zombies : le mimétisme, moyen exploré dans quelques exemples transgressifs du genre comme Shaun of the Dead (2004) et The Girl With All the Gifts (2016), où les personnages, pour peu qu'ils se donnent la peine de s'adapter à la façon d'être de la meute, parviennent à l'intégrer, c'est-à-dire à déambuler à ses côtés sans peur d'être dévorés. Plutôt que d'embrasser la condition zombie, c'est-à-dire le pouvoir de transcender les diktats dromocratiques qui régulent nos existences, les survivants tentent plutôt de trouver un " remède » à cette condition. Lexemple de World War $Z$ est encore utile ici, puisque le remède qu'y trouvent les scientifiques diégétiques nest pas vraiment un remède, mais un simple masque, une injection de pathogène mortel qui les dissimulent aux yeux des zombies, de sorte qu'ils puissent s'infiltrer subrepticement dans leurs rangs, à la manière des policiers en civil dans la meute des manifestants. En d'autres mots, il n'est jamais question pour les survivants de prendre exemple sur la meute ; ceux-ci n'ont d'autres choix que de l'affronter militairement ou de s'y dissimuler pour mieux la combattre, à l'instar d'ailleurs des représentants de lordre dromocratique actuel face à la meute prolétaire.

\section{Conclusion : manifestants zombies à l'heure du fascisme sécuritaire}

T a situation politique mondiale d'au_jourd'hui est particulièrement propice pour discuter des applications pratiques de la révolte zombie, alors que s'organisent simultanément l'attaque des mouvements de désobéissance civils dé-régulateurs tels qu'Extinction Rebellion, et la contre-attaque de forces policières de plus en plus militarisées. 
L'exemple parisien est éloquent, alors que c'est justement autour des questions de voirie que se clivent les parties en présence : les employés des transports en grève et les forces de répression étatiques, mais l'exemple d'Hong Kong demeure encore plus éloquent, ne serait-ce pour la bataille de voirie dantesque qui s'y déroule. À Hong Kong, les manifestants bloquent l'entrée aux bâtiments étatiques, utilisent le bitume des rues pour s'y laisser des messages, se meuvent par covoiturage improvisé, refusent les injonctions d'immobilisme, bloquent les aéroports et les stations de métro, brisent les machines distributrices de billets, recréant même une cartographie fluide de la ville pour identifier les trappes de police et les quartiers dangereux, cartographie fluide en parfaite adéquation avec le mantra nomadologique de Bruce Lee, ce fameux « be water ", mais aussi avec le pouvoir de pénétration et de lissage spatial de la meute zombie. De la meute prolétaire, qui, pour peu quelle refuse la tyrannie du mouvement productif, réussira certainement à vaincre l'état dromocratique une fois pour toutes.

\section{NoTES}

1. Kyle William Bishop, «The Idle Proletariat: Dawn of the Dead, Consumer Ideology, and the Loss of Productive Labor », The Journal of Popular Culture, Vol. 43, No. 2, 2010, p. 234.

2. Ibid., p. 234-235.

3. Ibid., p. 235.

4. Ibid., p. 238.

5. Sarah Juliet Lauro, «In a Supermarket at the End of the World» dans Zombie Theory: A Reader (éd. Sarah Juliet Lauro), University of Minnesota Press, 2017, p. 103.

6. Kyle William Bishop, op. cit., p. 241.

7. Paul Virilio, Vitesse et politique - Essai de dromologie, Éditions Galilée, Paris, 1977, p. 18.

8. Ibid., pp. 23-24.

9. Ibid., p. 18.

10. Ibid., p. 13.

11. Ibid., p. 83.

12. Ibid., p. 22.

13. Ibid., p. 18.

14. Gilles Deleuze et Félix Guattari, Capitalisme et schizophrénie II : Mille plateaux, Les Éditions de Minuit, Paris, 1980, p. 444.

15. Ibid., p. 456.

16. Ibid., p. 474. 